\title{
Abstract \#109351
}

\author{
Atomic Layer Deposition of Alumina on Silicon Nanotrees, Towards the Development of 3D \\ Ultrastable Aqueous Si Microsupercapacitor \\ A. Valero, D. Gaboriau (Univ. Grenoble Alpes, CEA, CNRS, INAC, SyMMES, Grenoble, CEA \\ Grenoble - INAC-PHELIQS (UGA, CEA)), A. Mery (Univ. Grenoble Alpes, CEA, CNRS, INAC, \\ SyMMES, Grenoble), P. Gentile (CEA Grenoble - INAC-PHELIQS (UGA, CEA)), and S. Sadki (Univ. \\ Grenoble Alpes, CEA, CNRS, INAC, SyMMES, Grenoble)
}

\section{Abstract Text:}

Key words: Microsupercapacitors, Silicon nanotrees, High-k dielectrics, aqueous electrolytes, PEDOTPSS.

In recent years, significant attention has been paid to the development of micro-devices as innovative energy storage solutions. For instance micro-sensor networks such as sensors actuators or implantable medical devices require power densities and cyclability that are several orders of magnitude higher than those of conventional Lithium-Ion batteries. For such applications, Microsupercapacitors (MSCs), a developing novel class of micro/nanoscale power source are rising alternatives, and their integration "on-chip" could allow significant innovations to emerge. ${ }^{1}$ Therefore, a great deal of attention has been focused on MSCs, for which large series of nanostructured active materials have been developed.

Following this trend, we have demonstrated through comprehensive investigations the interest of silicon nanostructures grown by Chemical Vapor Deposition (CVD) as electrodes materials for MSCs using ionic liquid electrolytes ${ }^{2,3}$. The fine morphological tuning of the nanostructure allowed by the bottom-up approach enables specific designs of electrode architectures, with a considerable leeway compared to other techniques. Such latitude allows optimizing porosity and ionic and electronic pathways while keeping robust mechanical and thermal performances, depending on the target application. Nanostructures such as SiNWs and SiNTrs have displayed excellent electrochemical performances being stable over more than 1 million cycles of galvanostatic charge/discharge under a $4 \mathrm{~V}$ wide electrochemical windows in EMI-TFSI ionic liquid, with large power densities of $10 \mathrm{~mW} . \mathrm{cm}^{-}$ 2 and good capacitance values of $0.5 \mathrm{mF} . \mathrm{cm}^{-2}$ at high current density of $0.5 \mathrm{~mA} . \mathrm{cm}^{-2} .4$

However a major silicon weakness which was still hindering its use with acqueous electrolytes is the native uncontrolled growth of silica when subjected to ambient atmosphere. Here we have developed a highly conformal passivation coating of a nanometric high-k dielectric layer of $\mathrm{Al}_{2} \mathrm{O}_{3}$ based on the rising Atomic Layer Deposition (ALD) technique. ALD has proven to allow a nanometric thickness control of the deposited layer while being highly conformal and covering. Electrochemical stability performances in ionic liquid, were enhanced allowing symmetric 2 electrode devices to reach an unprecedented cell voltage of $5.5 \mathrm{~V}^{5}$, improving energy and maximum power densities compared to pristine nanostructured silicon. The cyclability was also largely enhanced, with only $3 \%$ capacitance fade after $10^{6}$ galvanostatic charge/discharge cycles at $4 \mathrm{~V}$, and no degradation even after several $10^{5}$ resilience cycles over a $5 \mathrm{~V}$ window ${ }^{5}$. Moreover, the protective alumina layer enables the use of aqueous electrolytes for nanostructured Si based MSCs, which significantly increases the specific power of the devices up to $200 \mathrm{~mW} . \mathrm{cm}^{-2}$ at $0.5 \mathrm{~mA} . \mathrm{cm}^{-2}$ while keeping the capacitance performances at $0.5 \mathrm{mF} . \mathrm{cm}^{-2}$. Furthermore the system is remarkably able to retain $99 \%$ of its initial capacitance after 2 billion galvanostatic charge-discharge cycles at high current density of $0.5 \mathrm{~mA} . \mathrm{cm}^{-2}$ in an aqueous electrolyte of $\mathrm{Na}_{2} \mathrm{SO}_{4}$.

Eventually we have investigated the pseudocapacitive response of such MSCs in aqueous eletrolytes by a simple drop-cast method of a PEDOT-PSS film. The device exhibited promising performances with a specific energy of $2 \mathrm{Wh} . \mathrm{kg}^{-1}$ and a power density of $300 \mathrm{~W} . \mathrm{kg}^{-1}$ at a current density of $1 \mathrm{~A}^{-g^{-1}}$. The MSCs was able to retain $80 \%$ its initial capacitance after 50,000 galvanostatic charge-discharge cycles at $0.5 \mathrm{~A} \cdot \mathrm{g}^{-1}$. 
[2]Thissandier, F. ; Gentile, P. ; Pauc, N. ; Brousse, T. ; Bidan, G. ; Sadki, S. Nano Energy 2014, 5 , 20-27

[3]Thissandier, F. Gentile, P. Sadki, S., 2014, Journal of Power Sources 269, 740-746

[4]Gaboriau, D. Aradilla, D. Gentile, P. Sadki, S., RSC Advances, 2016, 6, 81017-81027

[5]Dorian Gaboriau, Maxime Boniface, Anthony Valero, Dmitry Aldakov, Thierry Brousse, Pascal Gentile, and Said Sadki

Acknowledgements 
The authors would like to thank the DGA, Direction General de l'Armement, and the CEA for their financial and technical support throughout the PhD thesis of A. Valero. 
1)

-Pristine NTs -Alumina protected NT

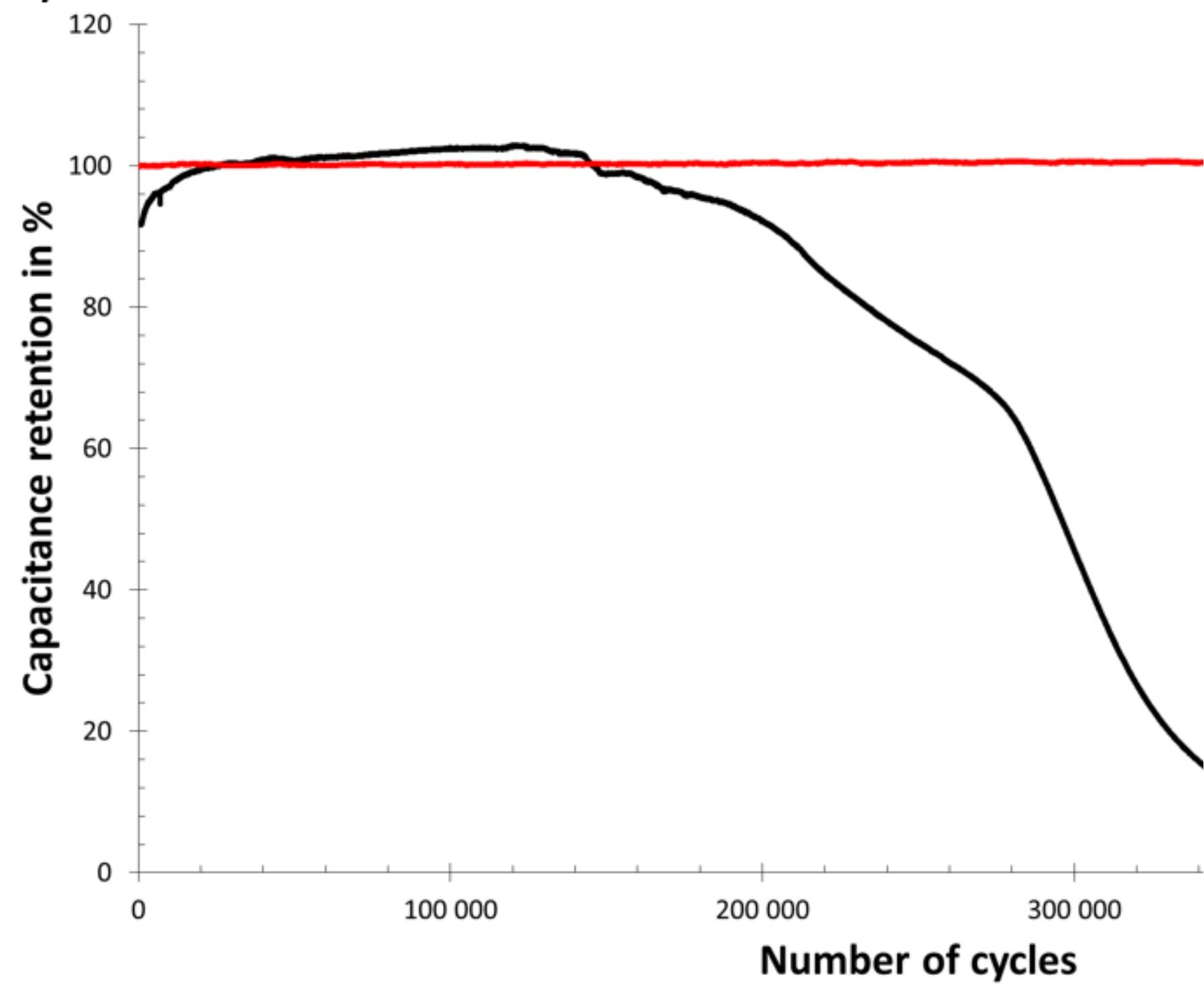

Fig. 1 Cycling performances of Alumina protected Si I in $\mathrm{Na}_{2} \mathrm{SO}_{4}$ at a current density of $0.5 \mathrm{~m}$

2)

-0 -Pristine Nts $\quad-0$-Alumina Protecte

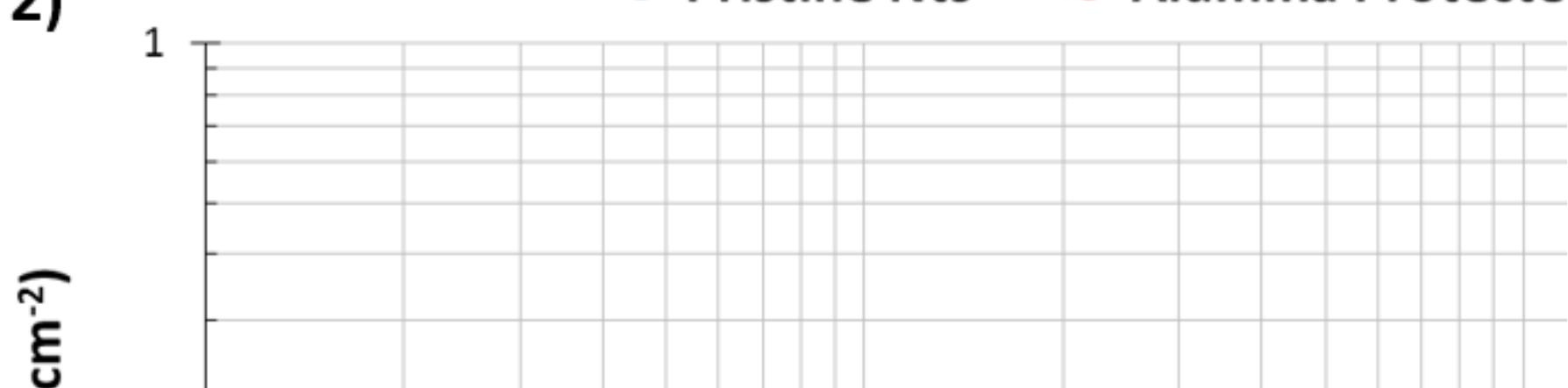

